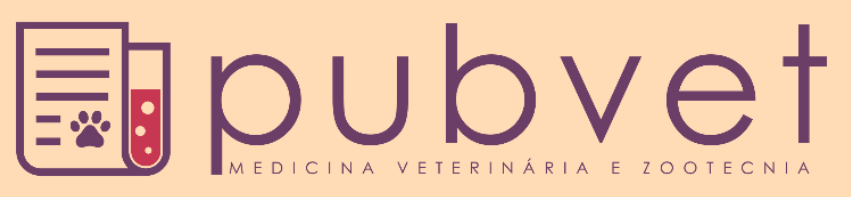

https://doi.org/10.31533/pubvet.v14n3a524.1-5

\title{
Criptococose felina: relato de caso
}

\author{
Julia Daniel Damiani ${ }^{1^{*}}{ }^{\bullet}$, Amanda Flores Teixeira ${ }^{1} \bullet$, Daniel Pereira dos $\operatorname{Santos}^{1}{ }^{\bullet}$, Lívia \\ Gonçalves da Silva Valente ${ }^{2} \theta$, Luciane Orbem Veronezi ${ }^{2}{ }^{\circ}$, Guilherme Valente de Souza ${ }^{2} \theta$ \\ ${ }^{l}$ Acadêmica de Medicina Veterinária, Centro Universitário Barriga Verde - UNIBAVE. Orleans - SC, Brasil. \\ ${ }^{2}$ Médico Veterinário, Docente, Centro Universitário Barriga Verde-UNIBAVE. Orleans - SC, Brasil. \\ *Autor para correspondência, E-mail: julia_damiane@hotmail.com
}

Resumo. A criptococose é uma micose sistêmica de distribuição cosmopolita causada por um fungo do gênero Cryptococcus, que acomete principalmente felinos. Embora seja considerada em felinos, é descrita raramente. Um felino, macho, SRD foi atendido no Hospital Veterinário do Unibave - HVU com histórico de dificuldade respiratória, nódulo ulcerado e deformidade na região dorso nasal, apresentando descargas nasais serosanguinolentas. O diagnóstico da criptococose se deu pela anamnese, exame clínico e citológico com observação do agente Cryptoccoccus neoformans. Foi estabelecido terapia com itraconazol durante seis meses, com regressão total do quadro e sem reação ao medicamento utilizado. $\mathrm{O}$ objetivo do trabalho foi relatar um caso de criptococose felina onde se obteve sucesso na terapêutica empregada.

Palavras chave: criptococose, felino, fungo, itraconazol

\section{Feline Cryptococcosis: case report}

\begin{abstract}
Cryptococcosis is a systemic mycosis of cosmopolitan distribution caused by a fungus of the genus Cryptococcus, which mainly affects felines. Although considered in cats, it is described as rare. A male feline SRD was treated at the Unibave Veterinary Hospital - HVU with a history of respiratory distress, ulcerated nodule and deformity in the nasal dorsum, presenting with bloody sero discharge. Cryptococcosis was diagnosed by anamnesis, clinical and cytological examination with observation of agent Cryptoccoccus neoformans. Itraconazole therapy was established for six months, with complete regression of the condition and no reaction to the drug used. The objective of this study was to report a case of feline cryptococcosis in which the therapy employed was successful.
\end{abstract}

Keywords: cryptococcosis, feline, fungus, itraconazole

\section{Criptococosis felina: reporte de un caso}

Resumen. La criptococosis es una micosis sistémica de distribución cosmopolita causada por un hongo del género Cryptococcus, que afecta principalmente a los felinos. Aunque se considera en los gatos, se describe raramente. Un gato criollo macho fue tratado en el Hospital Veterinario Unibave - HVU con antecedentes de dificultad respiratoria, nódulo ulcerado y deformidad en el dorso nasal, con secreción nasal serosanguinolenta. La criptococosis se diagnosticó mediante anamnesis, examen clínico y citológico con observación del agente Cryptoccoccus neoformans. La terapia con itraconazol se estableció durante seis meses, con una regresión completa de la afección y sin reacción al medicamento utilizado. El objetivo de este estudio fue informar un caso de criptococosis felina en el que la terapia empleada fue exitosa.

Palabras clave: criptococosis, felino, hongo, itraconazol 


\section{Introdução}

A criptococose é uma micose sistêmica de distribuição cosmopolita causada por um fungo do gênero Cryptococcus, que acomete principalmente felino, podendo atingir também caninos, animais silvestres e humanos (Norsworthy et al., 2004; Stradioto, 2010). Embora considerada em felinos, é descrita raramente (Barth et al., 2008). A levedura causadora da criptococose é conhecida como Cryptoccoccus neoformans e tem sua espécie subdividida em outras três variedades e cinco sorotipos que se diferem quanto à apresentação clínica, habitat e características epidemiológicas, sendo eles: $C$. neoformans var. grubii (sorotipo A), C. neoformans var. neoformans (sorotipos D e AD) e C. neoformans var. gattii (sorotipos B e C) (Franzot et al., 1999; Hnilica \& Medleau, 2012).

Segundo Pedroso \& Cândido (2006), no homem e nos animais as duas principais espécies que causam a doença são $C$. neoformans e $C$. gattii. Ambas as espécies são encontradas no ambiente associados a excrementos de pombos, material vegetal em decomposição e em tocos de árvores (Nelson \& Couto, 2015), onde pode permanecer viável por mais de dois anos (Marcasso et al., 2005). Reolon et al. (2004) relatam que as excretas envelhecidas de aves oferecem um substrato orgânico mais favorável para o desenvolvimento do fungo, pois possui menor quantidade de bactérias e, desta forma, a competição pelo crescimento é reduzida, contribuindo assim para a elevada densidade populacional de células de $C$. neoformans neste substrato.

Os felinos se infectam a partir da inalação dos esporos do C. neoformans, atingindo principalmente trato respiratório superior, podendo progredir para os pulmões. A disseminação via hematógena ou linfática, permite acometimento do sistema nervoso central (Honsho et al., 2003; Larsson et al., 2003; Nelson \& Couto, 2015; Reusch, 2010).

A infecção no trato respiratória dos gatos ocasiona sinais clínicos como descarga nasal serosa ou sanguinolenta, deformidade e oclusão nasais, rinite, espirro, sinusite e lesões oro nasais com presença de crostas ou úlceras, assim como ruídos respiratórios (Beatty et al., 2000; Norsworthy et al., 2004). Pereira \& Coutinho (2003) e Ettinger et al.(2017) descrevem o aparecimento de lesões com formação de massas firmes ou pólipos no tecido subcutâneo, principalmente na região nasal, conferindo um aspecto característico de "nariz de palhaço". Acontecendo o acometimento do sistema nervoso central os sintomas a serem observados incluem convulsões, paresia, ataxia, depressão e cegueira (Kerl, 2003). O diagnóstico pode ser feito através do histórico clínico do animal, anamnese, exames complementares como citológicos, histológicos, sorológico e cultura fúngica (Coelho et al., 2010).

\section{Relato de caso}

Foi atendido no Hospital Veterinário do Unibave - HVU um felino, macho, SRD, não castrado, pesando $3,1 \mathrm{~kg}$, de 5 anos de idade apresentando um nódulo ulcerado e deformidade na superfície dorsal nasal com aproximadamente $3 \mathrm{~cm}$ de diâmetro, secreção nasal serosanguinolenta e dificuldade respiratória (Figura 1).

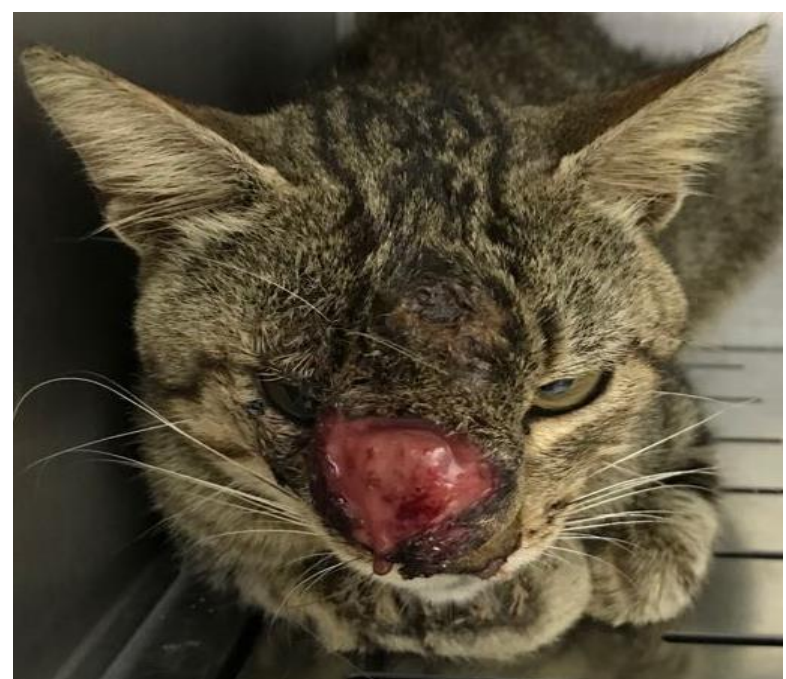

Figura 1. Paciente com lesões sugestivas de criptococose. 
Na primeira consulta ocorrida no dia 22 de janeiro de 2018, a proprietária relatou que a lesão havia iniciado há dois anos. O animal apresentava normúria, normodipsia, normoquesia e normorequixia. A partir do histórico e dos sinais clínicos suspeitou-se de granuloma fúngico (causado por um fungo do gênero Cryptococcus), com diagnósticos diferenciais de esporotricose e neoplasias cutâneas. Para diagnóstico definitivo realizou-se citologia por imprint e por punção aspirativa por agulha fina (PAAF) do nódulo.

$\mathrm{Na}$ avaliação citológica observaram-se inúmeras estruturas em formato de levedura, em alguns campos as leveduras apresentavam cápsula grande e clara apresentando brotamento com base estreita, além de quantidade moderada de eritrócitos, células inflamatórias polimorfonucleares (predominantemente neutrófilos), células mononucleares (com predomínio de macrófagos), restos e debris celulares, associados a algumas células epiteliais (Figura 2).
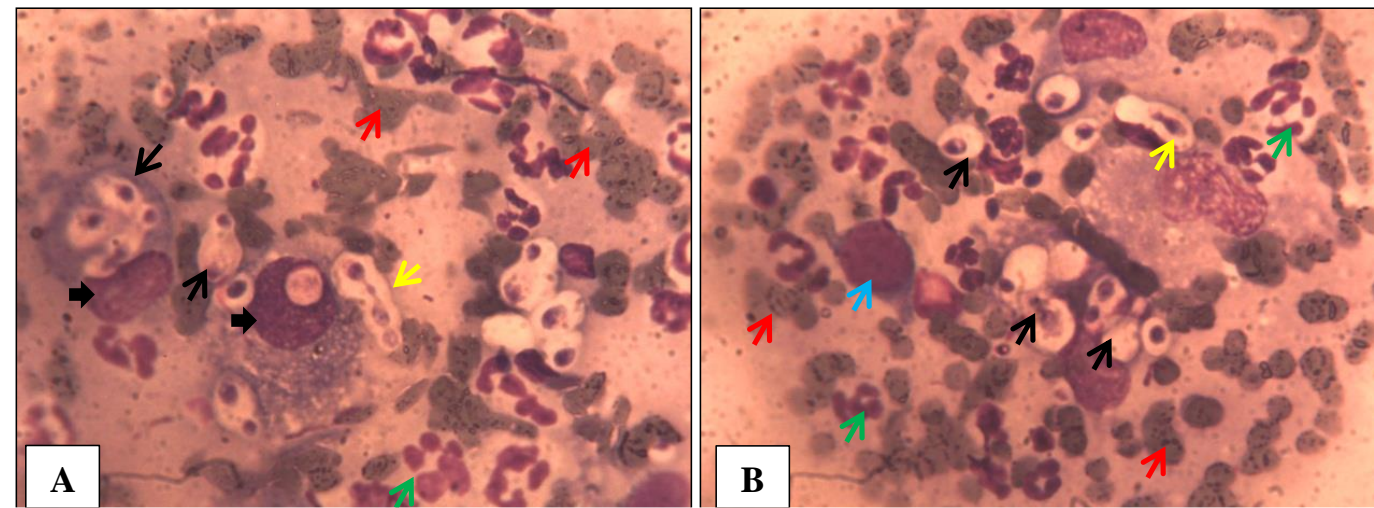

Figura 2. Criptococose. Verificam-se alguns microrganismos Cryptococcus caracterizados pela presença de levedura de tamanho médio com cápsula clara, associados a neutrófilos (setas verdes) e macrófagos (setas pretas grossas). Na imagem $\mathbf{A}$ veem-se algumas leveduras no citoplasma de macrófagos (setas pretas grossas). Em A e B; visualizam-se estruturas leveduriformes com cápsula grande e clara, apresentando brotamento de base estreita (setas amarelas). Adicionalmente verificam-se algumas células epiteliais (seta azul). O esfregaço possui um fundo de lâmina claro e rosáceo composto por inúmeros eritrócitos íntegros e degenerados (setas vermelhas). Fonte: Laboratório de histopatologia do HVU (2018).

Instituiu-se terapia antimicótica via oral com itraconazol 30mg, administração de um comprimido SID, e pomada de uso tópico Quadriderm ${ }^{\circledR}$ (valerato de betametasona + sulfato de gentamicina + tolnaftato + clioquinol) SID, por seis meses.

No primeiro retorno, no dia 26 de fevereiro de 2018, a lesão havia regredido consideravelmente (Figura 3), e ao fim do tratamento de seis meses o felino recebeu alta médica com total regressão da lesão apresentando-se completamente hígida e com aumento do peso corporal (Figura 4).

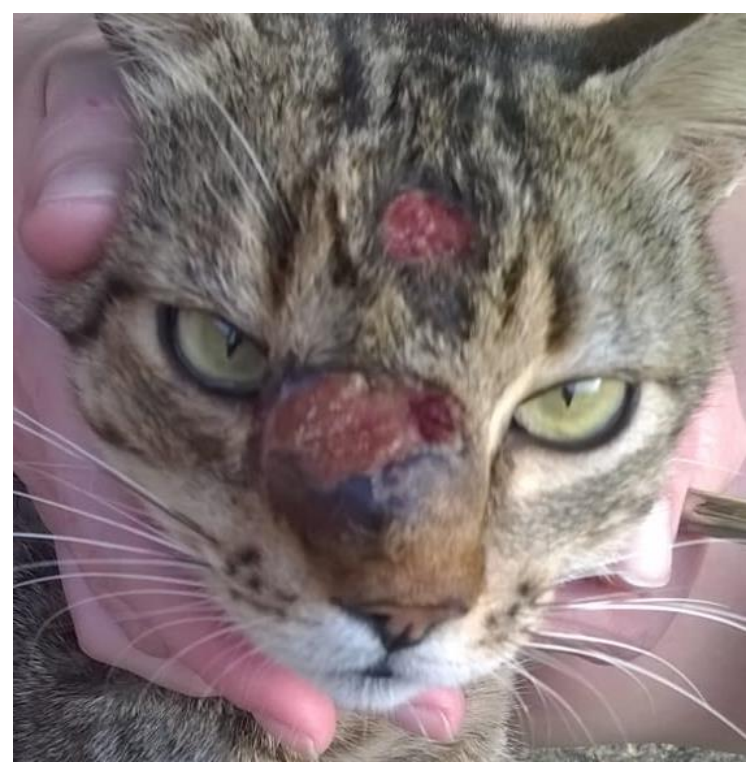

Figura 3. Paciente após 4 dias de tratamento, com a lesão apresentando regressão.

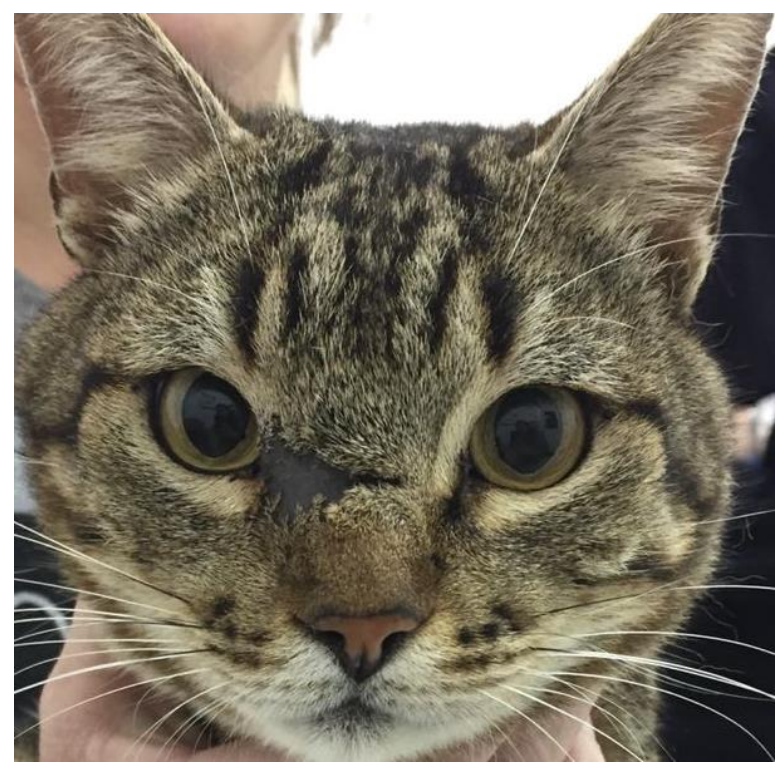

Figura 4. Paciente ao final do tratamento 


\section{Resultados e discussão}

O presente relato se justifica pela baixa casuística de criptococose felina descrita em literatura, ao passo de essa ser considerada uma patologia comum na clínica de pequenos animais (Barth et al., 2008). Como descrito por Martins et al. (2008), a maioria dos animais acometidos estava entre a faixa etária de 2 a 4 anos. O período de incubação da criptococose varia de meses a anos (Pennisi et al., 2013). Portanto o animal descrito, com cinco anos de idade, que já estava acometido há mais de dois anos adquiriu a doença no período etário descrito em literatura. Devido ao grande tempo de incubação torna difícil mensurar onde o animal adquiriu a infecção (Martins et al., 2008).

A sintomatologia observada no animal foi a mesma descrita em literatura quando aos quadros respiratórios e cutâneos, onde as nodulações ulceradas com deformidade na região dorso nasal observadas são descritas como sinal clínico clássico de "nariz de palhaço" (Ettinger et al., 2017; Pereira \& Coutinho, 2003). O quadro respiratório apresentado pelo felino atendido de descarga nasal sero sanguinolenta e respiração ruidosa também são algumas das características clínicas descritas por (Beatty et al., 2000).

O uso de citologia para diagnóstico de criptococose é um método fácil e eficiente para o diagnóstico definitivo, isso se dá devido à quantidade de leveduras, que geralmente é alta na lesão, e pela aparência característica do fungo Cryptococcus. Dois métodos eficazes citados para coleta de amostra foram utilizados neste relato, sendo eles imprint da lesão cutânea ulcerada e PAAF do nódulo (Bemis et al., $\underline{2000)}$.

Alguns dos protocolos terapêuticos a serem utilizados podem ser anfoterecina $\mathrm{B}$, cetoconazol. fluconazol ou itraconazol, este último sendo o de eleição para o presente relato, a média de tempo do tratamento com a droga é de 9 meses, podendo ser adotado protocolos de 3 a 24 meses (Pennisi et al., 2013). O itraconazol é considerado uma fármaco antifúngico efetivo, o que se mostrou verdadeiro com a total regressão do quadro do paciente em seis meses (Norsworthy et al., 2004). Segundo Stradioto, (2010) $\mathrm{m} 5 \mathrm{mg} / \mathrm{kg}$ de itraconazol já havia se mostrado efetiva no tratamento de criptococose .

\section{Considerações finais}

O diagnóstico por citologia foi de extrema importância para resolução do caso do felino, assim como a terapia com itraconazol que gerou total regressão do quadro mesmo este tendo se iniciado há bastante tempo antes de serem recorridos os serviços médicos veterinários por parte do tutor.

\section{Referências bibliográficas}

Barth, R. R. da S., Bruch, W. S., Castro, R. C. C., \& K.M.V., H. (2008). Levantamento prospectivo da ocorrência de Cryptococcus neoformans nas narinas de gatos portadores ou não de doenças respiratórias atendidos no hospital veterinário e fazenda escola da Faculdade comunitária de campinas-unidade III e região de campinas. Anuário Da Produção de Iniciação Científica Discente, $11,1-12$.

Beatty, J. A., Barrs, V. R., Swinney, G. R., Martin, P. A., \& Malik, R. (2000). Peripheral vestibular disease associated with cryptococcosis in three cats. Journal of Feline Medicine and Surgery, 2(1), 29-34.

Bemis, D. A., Krahwinkel, D. J., Bowman, L. A., Mondon, P., \& Kwon-Chung, K. J. (2000). Temperature-sensitive strain of Cryptococcus neoformans producing hyphal elements in a feline nasal granuloma. Journal of Clinical Microbiology, 38(2), 926-928.

Coelho, H. E., Moura, L. R., Orpinelli, S. R. T., Kock, G. P., Machado, F. M. E., \& Francisco Júnior, A. (2010). Cryptococcus neoformans associado à Meningite Felina em Uberaba, MG: Relato de caso. Veterinária Notícias, 15(1), 29-34.

Ettinger, Stephen J, Feldman, E. C., \& Cote, E. (2017). Textbook of Veterinary Internal Medicine-eBook. Elsevier Health Sciences.

Franzot, S. P., Salkin, I. F., \& Casadevall, A. (1999). Cryptococcus neoformans var. grubii: separate varietal status for Cryptococcus neoformans serotype A isolates. Journal of Cinical Microbiology, $37(3), 838-840$. 
Hnilica, K. A., \& Medleau, L. (2012). Dermatologia de pequenos animais: atlas colorido e guia terapêutico. Roca.

Honsho, C. S., Mine, S. Y., Oriá, A. P., Benato, N., Camacho, A. A., Alessi, A. C., \& Laus, J. L. (2003). Generalized systemic cryptococcosis in a dog after immunosuppressive corticotherapy. Arquivo Brasileiro de Medicina Veterinária e Zootecnia, 55(2), 155-159.

Kerl, M. E. (2003). Update on canine and feline fungal diseases. The Veterinary Clinics of North America. Small Animal Practice, 33(4), 721-747.

Larsson, C. E., Otsuka, M., Michalany, N. S., Barros, P. S. M., Gambale, W., \& Safatle, A. M. V. (2003). Canine ocular cryptococcosis: a case report. Arquivo Brasileiro de Medicina Veterinaria e Zootecnia (Brazil), 55(5), 533-538.

Marcasso, R. A., Sierra, S., Arias, M. V. B., Bracarense, A. P. F. R. L., Yamamura, A. A. M., de Biasi, F., Lopes, B. A., Amude, A. M., \& Cortêz, D. E. A. (2005). Criptococose no sistema nervoso de cães-relato de três casos. Semina: Ciências Agrárias, 26(2), 229-238.

Martins, D. B., Barbosa, A. L. T., Cavalheiro, A., Lopes, S. T. dos A., Santurio, J. M., Schossler, J. E., \& Mazzanti, A. (2008). Diagnosis of canine cryptococcosis by fine-needle aspiration cytology. Ciência Rural, 38(3), 826-829.

Nelson, R. W., \& Couto, C. G. (2015). Medicina interna de pequenos animais (Issue 1). Elsevier Editora.

Norsworthy, G. D., Crystal, M. A., Grace, S. F., \& Tilley, L. P. (2004). O paciente felino. São Paulo: Roca, 3, 300.

Pedroso, R. S., \& Cândido, R. C. (2006). Diagnóstico laboratorial da criptococose. News Lab, 14(77), 94-100.

Pennisi, M. G., Hartmann, K., Lloret, A., Ferrer, L., Addie, D., Belák, S., Boucraut-Baralon, C., Egberink, H., Frymus, T., \& Gruffydd-Jones, T. (2013). Cryptococcosis in cats: ABCD guidelines on prevention and management. Journal of Feline Medicine and Surgery, 15(7), 611-618.

Pereira, A. P. C., \& Coutinho, S. D. A. (2003). Criptococose em cães e gatos-revisão. Clínica Veterinária, 45, 24-32.

Reolon, A., Perez, L. R. R., \& Mezzari, A. (2004). Prevalence of Cryptococcus neoformans in urban pigeons of Porto Alegre (RS), Brazil. Jornal Brasileiro de Patologia e Medicina Laboratorial, 40(5), 293-298.

Reusch, C. (2010). Feline diabetes. In S J Ettinger \& E. C. Feldman (Eds.), Textbook of veterinary internal medicine (pp. 1796-1816). Saunders.

Stradioto, E. A. P. (2010). Criptococose felina: relato de caso e revisão bibliográfica. In Monografia. Universidade Castelo Branco.

Recebido: 19 de novembro, 2019.

Aprovado: 17 de dezembro, 2019.

Publicado: 21 de abril, 2020.

Licenciamento: Este artigo é publicado na modalidade Acesso Aberto sob a licença Creative Commons Atribuição 4.0 (CC-BY 4.0), a qual permite uso irrestrito, distribuição, reprodução em qualquer meio, desde que o autor e a fonte sejam devidamente creditados. 